\title{
The dark side of microrheology: non-optical techniques
}

\author{
Giovanni Vleminckx Christian Clasen*
}

November 3, 2014

\begin{abstract}
Microrheology probes the mesoscale between bulk rheology, which provides an integral sample response, and nanorheology, which refers to a local response at a molecular confinement level. The term 'microrheology' is often used to refer to optical particle tracking methods that measure a local response of a sample. In contrast to this, non-optical microrheology techniques generally allow two different effects to be studied: actual confinement effects on the rheology and boundary effects such as slip. Investigating the mesoscale range has additional advantages such as the possibility to perform measurements with small sample volumes and at high shear rates. This review bundles the wide array of non-optical techniques into five categories: adaptations to a conventional rotational rheometer, sliding plate rheometry on a micrometer scale, microfluidics, piezo vibrators and radial channel flows. The concept of each set of techniques is described, together with their operational window and examples of recent studies.
\end{abstract}

\section{Introduction}

Rheology as the science of flow correlates the deformation and deformation rate history of a material to its state of stress, which is related to the underlying internal microstructure of the fluid or soft solid. Bulk rheological measurements probe an overall mechanical response of a sample on a macroscopic level and allow an interpretation of the results with respect to a known or assumed microstructure and its evolution. However, the integral response of a bulk measurement cannot offer any direct information on the microstructure, which can vary locally $[17,64]$. Moreover, bulk measurements fail to characterize the behavior of fluids under many processing conditions and usage applications that operate under a strong confinement of the fluid and its microstructure. Examples include extrusion, bearing lubrication, inkjet printing, blood flow through capillary veins, or oral processing of food [24]. Consequently, there is a need to rheologically probe shorter length scales. Molecular thin films of a material can be studied via techniques that have been referred to as 'nanorheology' [34, 66, 72]: atomic force microscopy (AFM) $[77,81]$, nanoindentation [96] or with the surface force apparatus (SFA) [54]. Although these approaches examine a local rheological response, they are aimed at probing an abnormal response of simple fluids on a molecular confinement level, often focussing on the physicochemical interactions with the confining substrate. In contrast to this, flows in a mesoscale range (roughly spanning dimensions of $100 \mathrm{~nm}-100 \mu \mathrm{m}$ ) are of rheological interest if the bulk flow itself is affected by a confinement. This is not the case for simple fluids but for complex fluids or soft matter with microstructural length scales comparable to the confinement (and thus for many industrial processing operations as well as in the field of microfluidics). This range cannot be explored using conventional bulk rheometry nor with nanorheology.

Microrheology aims at closing this gap with experimental research in the mesoscale region. There are in principle three classes of effects that are probed with microrheology: actual confinement effects that lead to a different rheological response of the whole sample, itself depending on the degree of confinement. An example of this would be a confinement induced jamming of particle suspensions, which has been reported to occur for separations between the shearing surfaces smaller than 5 times the particle diameter [5]. Secondly, microrheology can probe the local response of a heterogeneous sample. Such a local response (e.g. the medium viscosity in the mesh of a cross-linked hydrogel) can be different from the response of the bulk sample (the gel itself) but is not necessarily arising from a

\footnotetext{
*Department of Chemical Engineering, KU Leuven, Willem de Croylaan 46, 3001 Heverlee, Belgium, e-mail: christian.clasen@cit.kuleuven.be, phone: +32 (0) 16322354
} 


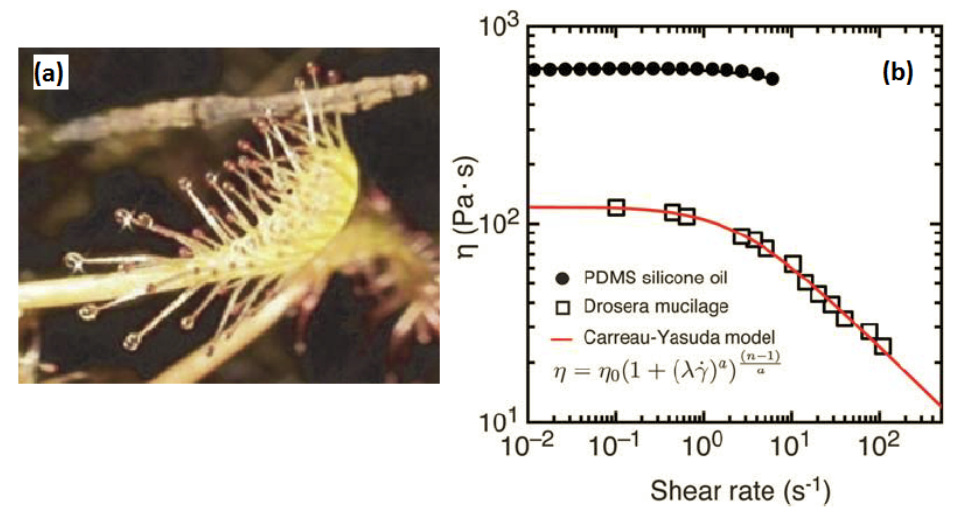

Figure 1: a. A single leaf of the Drosera plant with secreted mucilage drops. b. A flow curve of Drosera mucilage, measured with a flexure-based microgap rheometer on a sample volume of $0.3 \mathrm{~nL}$ [28].

confinement effect. Thirdly, microrheology enables the investigation of boundary effects such as wall slip or cohesive and adhesive failure. Such boundary effects are also inherently present in bulk flow investigations, but can often be neglected due to a dominating stress response of the bulk. However, in microrheological investigations, they happen on a similar scale as the global deformation of the bulk sample and can therefore be investigated with much higher resolution $[6,14,18]$. Beneficial side effects of some microrheological techniques are that they often require only very small fluid samples (as much as $50 \mu \mathrm{L}[78]$ or even below $10 \mu \mathrm{L}[17,28,53]$ ), facilitating the study of samples that are expensive or only available in limited quantities. Figure 1 gives an example of a flow curve obtained from a sample volume of only $0.3 \mathrm{~nL}$. Furthermore, decreasing the length scale over which a sample is probed allows to attain high shear rates [16] that are encountered in many industrial processes (e.g. $\dot{\gamma} \geq 10^{6} \mathrm{~s}^{-1}$ as reported in $[23])$.

The broad range of microrheological techniques that can be used to probe the mesoscale can be broken down into two categories: optical and non-optical techniques. Although initially used to describe all rheological investigations on a microscale, the term 'microrheology' is nowadays mainly recognized for passive as well as actively-driven particles and their, most of the time, optical tracking. Tracer particles are added to a material and their motion is analyzed to retrieve relevant rheological properties. A recent overview on optical microrheology applied in the field of food gels and emulsions has been presented by Moschakis covering topics as magnetic and optical tweezers or diffusing wave spectroscopy [64], and other comprehensive overviews have been presented by MacKintosh and Schmidt [57], by Squires and Mason [87] and by Waigh [93]. Although these approaches can have excellent correlation with bulk rheological data, it should be realized that optical microrheology probes a sample only locally. While this can enable one to separate the rheological response of a heterogeneous sample, care needs to be taken in the interpretation of results as for example the probe particles can interact with the surrounding sample, increasing the risk of erroneous results [12]. Furthermore, these optical microrheological techniques do not allow to probe confinement effects acting on the whole sample, which can, however, be probed with non-optical techniques.

This review article gives an overview of the extensive range of non-optical microrheological methods, subdivided into five categories. While focusing on a description of concept for each group, examples of specific techniques are provided together with their operating window. Recent studies are indicated that demonstrate the importance of these techniques for fundamental research, processing industry and fine-tuning consumer relevant properties.

\section{Techniques}

Despite the fact that there seems to be a trend of proliferation in different non-optical microrheological techniques, conceptual similarities arise that allow organization into five clusters. A first category consists of approaches that use commercially available rotational rheometers at narrow gaps. A second category are simple planar Couette flows in micrometer dimensions that are generated in sliding plate rheometers 


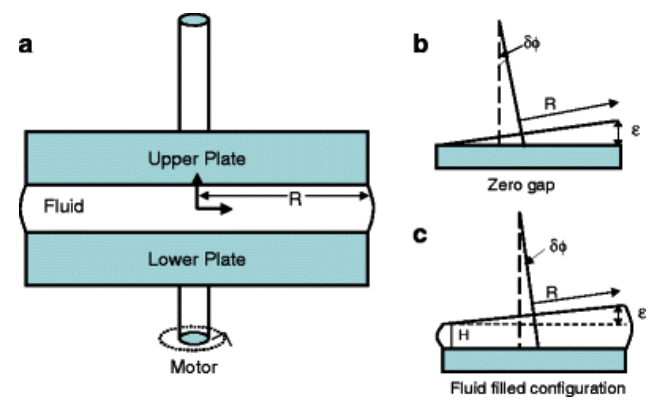

Figure 2: Illustration of a parallel plate geometry in a conventional rotational rheometer with $R$ the radius of the plates, $H$ the gap height, $\Omega$ the angular velocity, $\delta \phi$ the misalignment angle and $\varepsilon$ the gap error. a. shows the ideal situation without gap error while b. and c. show issue due to a gap error during zero gap determination and measurement respectively [75].

and their derivatives. A broad range of microfluidic devices make up a third group. The final two clusters contain more exotic devices that are distinct each in their own nature. Although each set of techniques has its own limitations or problems, one of the common measurement issues is the decreased applicability of the no slip boundary condition. A brief description of wall slip is therefore discussed together with other issues in the first cluster.

\subsection{Adaptations to a conventional rotational rheometer}

While different techniques have been developed to study the rheology of fluids under high shear or in microscale confinement, probably the most accessible route is the adaptation of commercially available rheometers. Reports on utilizing rotary parallel-plate rheometers operating at micrometer gaps go back to the early 80 's $[21,47,48]$ and the idea has been revisited frequently due to the simplicity of this approach and the ongoing technical improvements of commercially available rotational rheometers [24, $26,37,65,75]$. The different approaches can be categorized in two groups: identifying the possible errors that will arise when operating parallel-plates at micrometer gap separations (and correcting data for these errors and/or identifying the experimental limits), and secondly, improving the shear geometries in order to minimize the errors. The main errors that arise when setting small apparent gaps are both systematic errors in the commanded gap of the rheometer, as well as misalignment of the shearing surfaces towards each other (parallelism), the surface alignment towards the rotational axis of the rheometer (perpendicularity) and a possible bending or torsion of the surface itself (flatness) (it should be noted that the use of the terms parallelism and flatness in rheological literature is ambiguous and also used with regard to the alignment of the rotational axes). Regularly these errors are combined into a total gap error with which the apparent gap is corrected in order to determine the actual effective shear rate applied to a sample. Two recent publications by McKinley and co-workers [75] and Stokes and co-workers [24] deal in detail with the errors that arise when using a conventional rheometer in narrow gaps. Non-parallelism, as visible in figure 2, is considered the major contribution to the total gap error $\varepsilon$ and is typically found to be of the order of $5-50 \mu \mathrm{m}[24]$.

Improvements of the shear geometries to reduce the effect of non-flatness have been introduced by Mackay and co-workers. They showed that it is possible (with a very well-aligned rotational rheometer and specially-machined parallel plates) to perform steady shear experiments down to gaps of $10 \mu \mathrm{m}$ [37]. They also adjusted the flatness of the shearing surfaces by in situ surface lapping of the rotating parallel-plates e.g. with diamond paste, and simultaneously reducing the roughness below micron size. [56]. Stokes and co-workers recently presented a study [24] in which they evaluated the capability of commercially available rotary rheometers for accessing shearing gaps below $100 \mu \mathrm{m}$ with parallel plates that were, however, selected from a number of similar fixtures with regard to their perpendicularity error. With this they were able to achieve total gap errors as low as $\varepsilon=25 \mu \mathrm{m}$. Figure 3 shows their gap-dependent measurement results on a polymer solution and the corresponding gap error correction.

Stokes and co-workers also pointed out that an additional substantial gap error can be induced during the procedure applied to determine the zero gap setting of the plates [25]. The squeeze flow of air between well aligned plates can influence the normal force arising during the gap closing which is regularly used 

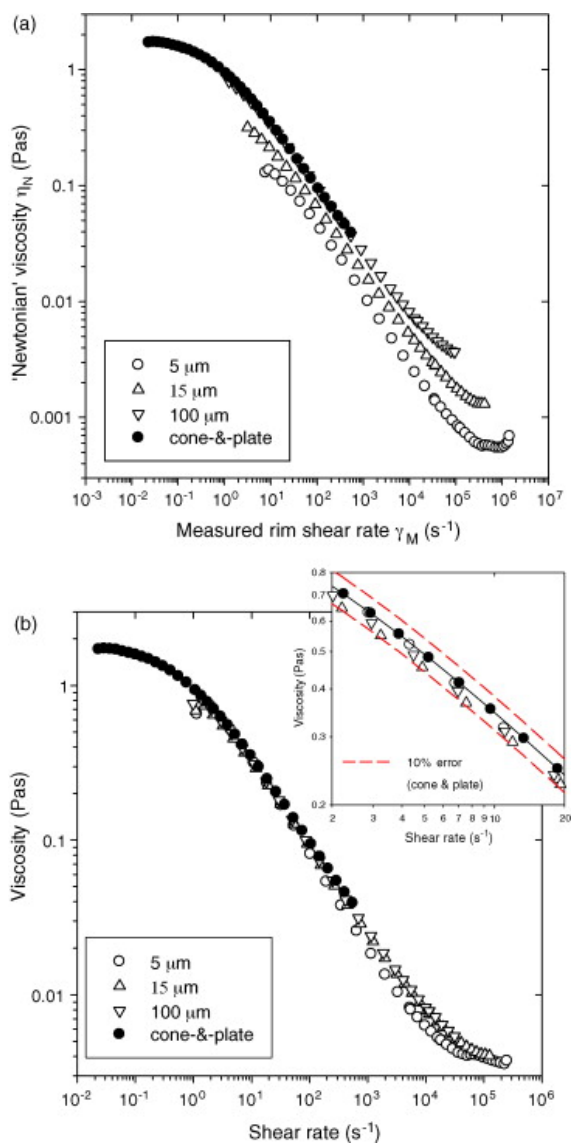

Figure 3: a. Flow curves of a $0.625 \%$ aqueous polyacrylamide solution measured in a conventional rotational rheometer with apparent gap separations from 5-100 $\mu \mathrm{m}$ by Davies and Stokes [24]. From this they determined a total gap error of $\varepsilon=25 \mu \mathrm{m}$. b. Correction of the measured viscosity and rates based on this total gap error. 
to determine the touching point of the shearing surfaces.

A number of recent studies have used the above mentioned adaptations and gap error corrections of parallel-plate setups with micrometer gap separations with a focus on studying fluids a high shear rates $[23,24,46,60,75]$, and on confinement effects $[23,24,46,89,95]$. In addition, these setups have been used to study small sample volumes [60], and to mimic usage or processing environment conditions $[23,46,89]$.

Several researchers have recently investigated the effects of non-parallelism, -flatness and -perpendicularity of the parallel plates on the normal stresses determined with rotational plates parallel-plates at small gap separations. The misalignment can cause a lubrication flow that results in an additional pressure in the small gap that scales (following the Reynolds equation) with the inverse gap to the third power. Both numerical and analytical solutions of the Reynolds equation have been utilized to correct for the lubrication stresses at small gaps and to still extract the rheological response of the fluid $[2-4,16]$. A recent parallel plate design utilizes the lubrication pressure to align the rotating parallel plates (of which one can continuously adjust its position) and minimize the parallelism error [15]. Although only operating at high rotation rates, this design was capable to measure at gap separations down to $10 \mu \mathrm{m}$, limited only by the non-flatness of the surfaces. Xie et al. [97] used a similar idea of self-aligning of tilting parallel disks via hydrodynamic lubrication, however, not in a rotating mode. They aim was to measure the pressure drop observed for the radial flow between the two disks in order to determine the high shear viscosity (the gap separations were limited to $>50 \mu \mathrm{m}$ ). A similarly exotic setup to minimize parallelism and flatness errors has been suggest by Gong and co-workers who introduced the idea to operate the parallel plate geometry of a rotational rheometer with the addition of elastic hydrogel surfaces. Lubrication flows and the arising normal forces deform the elastic surfaces and level out their misalignment $[35,40,41]$. They also suggested the use of osmotic pressure to actively control the gap separation between the deformable shearing surfaces.

A straightforward way to determine and correct for the gap errors is to assume all gap errors to be combined in an effective extra gap $\varepsilon$ that adds to the commanded gap $H$ so that the apparent shear rate $\dot{\gamma}_{a}=\Omega R / H$ (with $\Omega$ as the angular velocity) can be corrected to give the true shear rate $\dot{\gamma}_{t r u e}=\Omega R /(H+\varepsilon)$ [47]. Measuring the same fluid (for simplicity best a Newtonian one) at the same stress level but with different nominal gaps $H$ enables one to determine the total gap error $\varepsilon$ from

$$
\frac{1}{\eta_{a}}=\frac{1}{\eta_{\text {true }}}\left[1+\frac{\varepsilon}{H}\right]
$$

A linear regression of $1 / \eta_{a}$ versus $1 / H$ yields the reciprocal of the true viscosity $1 / \eta_{\text {true }}$ as the intercept and $\varepsilon / \eta_{\text {true }}$ from the slope. The quantified total gap error $\varepsilon$ (which is normally a systematic error of the used geometry and/or instrument) can then be used in further measurements to correct the data. An example of such a correction to study the true flow curve of a polyacrylamide solution at apparent gaps of $5-100 \mu \mathrm{m}$ is shown in a study by Davies et al. [24] (see figure 3).

It should be noted that the gap error is generally not induced by operating a device at micrometer gap separations, but in principle always present and only starts to dominate the apparent shear rate when the error reaches a similar dimension as the commanded gap $H$. A similar 'ever present' effect that gains more importance when operating at smaller gap settings is slip. Whereas for bulk measurements, no slip is a widely-accepted (and in many cases valid) boundary condition, decreasing the gap separation will eventually lead to a dominating effect of slip on the rheological response [85]. Similar to the gap error, slip is not caused by the small gaps, but rather occurs when a rheological experiment reaches sufficiently high stresses [99]. For a detailed discussion and review on wall slip we refer here to the work of Barnes [7], Buscall [9], Sochi [85] and Prud'homme [99]. Slip phenomena can be subdivided in two categories, i.e. true slip and apparent slip. True slip is considered to be present when there is a discontinuity of the velocity profile at the wall $[10,82]$. On the other hand, a localized region of a higher velocity gradient close to the wall is called apparent slip, e.g. observed for suspensions and emulsions. [62] Apparent slip can be caused by a variety of mechanisms [85]: depletion of the boundary layer at the wall [86], formation of a lubrication layer [62], or adsorption of a particle layer on the surfaces [83], adhesive failure at the interface [1], cohesive failure near the wall [94] or polymer chain disentanglement [55]. The no slip boundary condition is violated in case of true wall slip but not for apparent slip [7, 85]. Slip is commonly characterized by a slip velocity and a slip layer thickness. The slip velocity $V_{s}$ is the difference between the velocity of the wall and that of the last fluid layer near the wall that behaves as part of the bulk. At steady state, this velocity is a function of the stress only [99]. The thickness of the slip layer $\delta$ (so the 

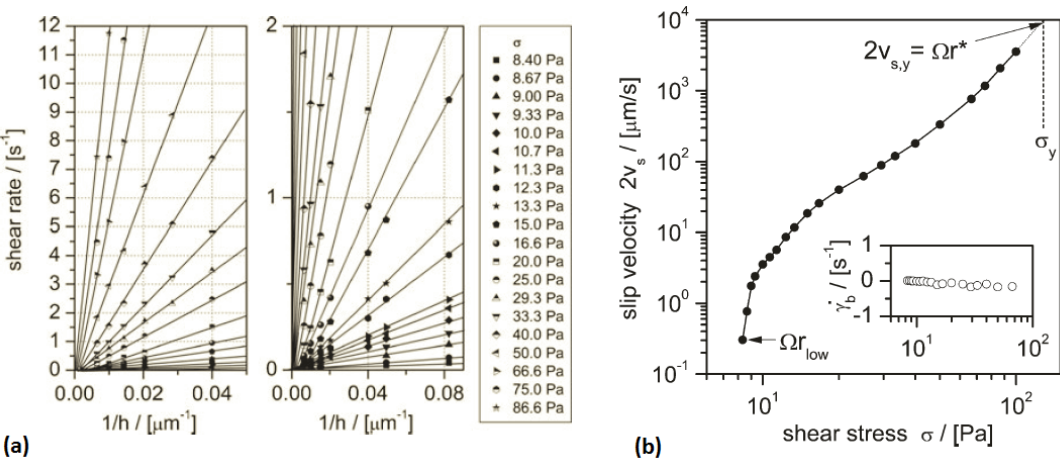

Figure 4: a. Mooney analysis of flow curve data of a $5 \mathrm{wt} \%$ aqueous xanthan gum solution obtained with shearing gap separations of $5-150 \mu \mathrm{m}$. Linear regression of data of the apparent shear rate as a function of the inverse gap (at a constant stress level) using equation 3 yields the bulk shear rate and slip velocity from the $y$-axis intercept and slope respectively. $\mathbf{b}$. The slip velocity as a function of the measured stress as determined from the Mooney analysis. The inset shows the bulk shear rate [14].

distance between the wall and the last bulk fluid layer) depends on the slip mechanism and has molecular dimensions for true slip, but can be on the order of $0.1-10 \mu \mathrm{m}$ for apparent slip mechanisms [7].

Wall slip can cause significant errors in rheological measurements (e.g. false thixotropy, erroneous yield stress detection) but the effects are often underestimated [9, 85]. As pointed out by Buscall [9], the fractional error $e$ on the viscosity caused by apparent slip, $\eta_{a} / \eta-1$, can be described in terms of the ratio of the slip layer thickness to the characteristic scale of the measurement geometry and on the relative viscosity of the system and is given by

$$
e=1-\frac{\eta_{a}}{\eta}=\frac{\eta \delta}{\eta_{\delta} H+\eta \delta}
$$

where $\eta_{\delta}$ is the viscosity of the slip layer liquid. Similarly, for true slip this error percentage can be formulated as $e=V_{s} /\left(\dot{\gamma} H+V_{s}\right)$. It is obvious that in both case the error induced by slip increases with decreasing gap. In rheological measurements on a microscale, wall slip is therefore generally not negligible. There are different techniques to eliminate wall slip as much as possible. In some methods, researchers have changed the physical nature of the solid boundaries by changing their surface roughness or physicochemical properties [76]. Furthermore, choosing a different kind of geometry is also possible, e.g. a vane geometry $[7,9,85]$ (although this is then not related to microrheology anymore). In recent microrheological studies, rather than attempting to reduce wall slip, researchers have utilized this increasing effect of slip with decreasing gap in order to study the slip mechanism in detail. This has been done for example to investigate the relation between the slip velocity and the shear stress for a yield stress fluid [14], or to relate slip in polymer melts to a microstructural source [36].

Such a characterization of the wall slip is traditionally done through rheological measurements at different sizes of the characteristic measurement scale for the construction of a Mooney plot [63, 99]. For this the apparent shear rate $\dot{\gamma}_{a}$ is plotted versus the reciprocal of different measurement scales $H$, but for a given constant shear stress $\sigma$. From

$$
\dot{\gamma}_{a}=\dot{\gamma}+\frac{V_{s}}{H}=\dot{\gamma}+\frac{\sigma \delta}{\eta_{\delta} H}
$$

one can extract the true shear rate $\dot{\gamma}$ from the y-axis intersect and the slope gives the slip velocity $V_{s}$ (for true slip) or the slip layer thickness $\delta$ (for apparent slip). The characteristic measurement scale depends on the measurement system, so for microrheology with parallel plates this is the varying gap setting, but it could also be for example the capillary diameter for a (micro-)capillary rheometer [7, 9, 85, 99]. A recent example of such a slip analysis, obtained from measurements with absolute micrometer gap settings from 4.5 to $100 \mu \mathrm{m}$ is given in figure 4 .

It should be noted that it is principally not possible to differentiate between the effects of wall slip and gap error on parallel plate (micro-)rheometry. Equations 1 and 3 use the same fitting procedure of plotting apparent rheological parameters as a function of the inverse gap. While (luckily) both give 
the sought true rheological parameters from extrapolation, it also means that one cannot distinguish the cause of the gradient in equations 1 and 3. Unfortunately most of the literature on parallel plate microrheology is discussed only in terms of either one of the two possible error sources.

In addition to the aforementioned errors, additional effects can arise when measuring with parallel plates at micrometer gap separations. An overview on general errors that are difficult to correct for can be found in [24]. For these errors, where no corrections are possible, the window of operation in which rheological measurements can be trusted needs to be known in order to judge the quality of a measurement.

One of these error sources is viscous heating. The temperature of the sample can increase when being sheared through the dissipation of energy as heat. At high shear rates and for highly viscous fluids this can lead to a decrease in viscosity with time. Although viscous heating is not necessarily limited to microgap rheometry, the high deformation rates that can be achieved when approaching small gap separations increase the chance of viscous heating. On the other hand the small sample volume encountered in narrow gap rheometry enables a fast heat dissipation to the confining surfaces. The competition of these effects can be captured by the Nahme number that describes the ratio of the time scales for thermal diffusion to viscous heating:

$$
N a=\frac{\eta \beta H^{2} \dot{\gamma}_{\text {true }}^{2}}{k T}
$$

where $k$ is the thermal conductivity, $T$ is the temperature and $\beta=d \eta / d T^{T} / \eta$ is the thermal sensitivity of the sample [75]. At low values of $N a$, viscous heating is negligible. The Nahme number increases quadratically with both shear rate and gap height. For parallel plate experiments with $\dot{\gamma} \sim 1 / H$ the Nahme number is therefore independent of the gap setting for a given angular velocity.

Edge effects are of general concern for parallel plate rheometry. The sample shape in between the parallel plates that is ideally cylindrical can differ at higher shear rates. However, edge effects on the measured viscosity are of the order $O(H / R)^{4}[69]$ and become therefore negligibly small in narrow gaps. $[75]$

Similarly, radial migration (and sample loss), due to centrifugal stresses that occur above a critical shear rate, becomes of less importance when approaching small gaps. The critical rate increases with decreasing gap $\left(\dot{\gamma}_{c} \sim H^{-3 / 2}\right)$, the effect of centrifugal stresses reduces thus with decreasing gaps. [24, 75]

Finally, an apparent increase in viscosity can be observed as a result of secondary flows. Significant increases in viscosity of Newtonian fluids have been measured when the Reynolds number is getting large:

$$
R e=\frac{\rho \dot{\gamma} H^{2}}{\eta}
$$

where $\rho$ is the density [24]. A critical value of $R e=100$ has been reported by Turian for this [90]. Again, for decreasing gaps the value of angular velocity at which this critical value of $R e$ is reached increases so that secondary flow becomes less important when approaching micrometer gap separations.

The awareness of the above mentioned limitations and possible errors has led to a number of recent studies that have shown the feasibility of using parallel plates at gap spacings ranging from $1000 \mu \mathrm{m}$ down to $5 \mu \mathrm{m}$. Crawford et al. [23] studied the agglomeration of particles in chemical mechanical polishing slurries, which are used in the semiconductor manufacturing industry to polish electronic materials. Furthermore, in the growing research field of food design, rheology of thin films is becoming increasingly important to correlate food microstructure and composition on one hand and sensory perception on the other hand $[46,89]$. The shear rates that are achieved with parallel plate microrheometry can be as high as $10^{5} \mathrm{~s}^{-1}$ and a number of publications focussed on this 'side effect' of microrheometry studying the high shear behavior of fluids $[23,24,46,56,60,75,89]$.

\subsection{Sliding plate rheometry on the micrometer scale}

Similar to rotating parallel plates a sliding plate rheometer can in principle be operated as a microrheometer by decreasing the gap separating the shearing surfaces. Typically a sliding plate rheometer consists of two parallel surfaces that move with a translational velocity relative to each other to create a planar Couette flow (as illustrated in figure 5), although the mechanics to move the surfaces and retrieve a response differ between techniques [33]. Sliding plate rheometers have recently been used to study the 


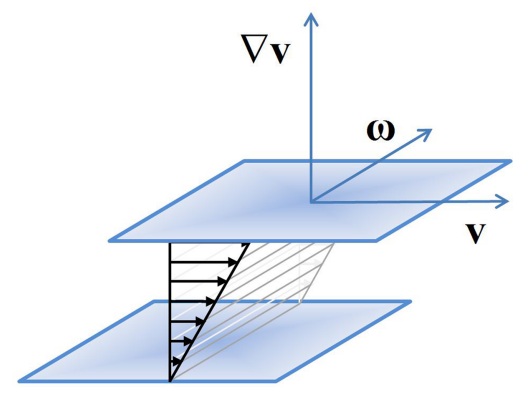

Figure 5: Planar Couette flow between two parallel surfaces moving with a velocity relative to each other [74].

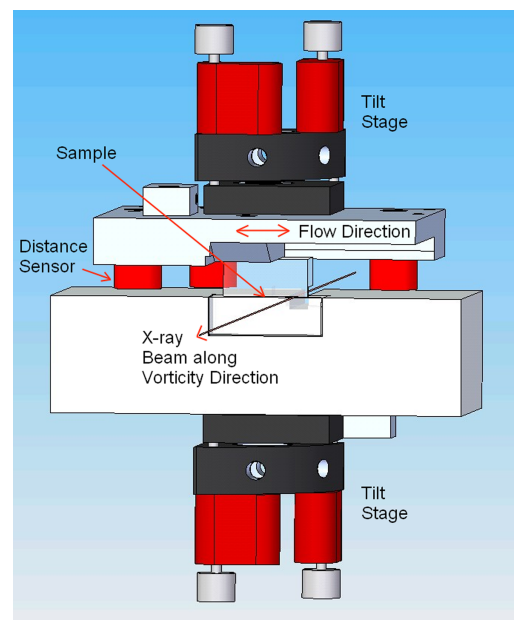

Figure 6: CAD rendering of the flow cell of the FMR micro gap rheometer. Two optical flat glass surfaces confine the sample. The micrometer gap between the shearing surfaces is aligned using nanopositioning tilt stages and controlled by non-contact distance sensors [74].

gap-dependent behavior in microstructured multiphase fluids $[17,52,74]$ or for indirect measurements of the slip velocity of fluids $[14,36]$.

In contrast to the simple adaptations to rotational rheometers, distinctively different types of sliding plate rheometers are used. A first type, based on the rheometer designed by Dealy and co-workers [33], is the flexure-based microgap rheometer (FMR) constructed by the groups of Clasen and McKinley $[6,14,17,18]$. The FMR develops a planar Couette shearing flow (Figure 5) between two optical flats (Figure 6 shows a schematic of the flow cell), which are each attached to a set of independent compound leaf springs. The gap between the shearing surfaces can be controlled with nano-actuators over a range of $1-150 \mu \mathrm{m}$. Non-contact inductive proximity sensors are used to monitor the gap, the parallelism of the surfaces and the shear rate and stress via the displacement of the two springs. Additionally, the second generation of the FMR, allows simultaneous measurement of the first normal stress difference $N_{1}$ up to $25 \mathrm{kPa}[6]$.

Similar to the FMR, a biaxial shearing cell with a gap separation of $9 \mu \mathrm{m}$ has recently been developed by Cohen and co-workers in combination with a confocal microscopy setup [52]. A multi-axis piezo allows movement of one shearing surface along all directions while the fluid response is measured by a biaxial force measurement device (FMD) attached to the second surface. The FMD can reliably measure shear stresses over the range of $0.05-2000 \mathrm{~Pa}$.

A third type of sliding plate rheometer was recently designed to extract the storage and loss moduli of complex fluids confined between two sliding plates. Based on earlier developments as the oscillatory sliding plate rheometer by Granick and coworkers [72] and Braithwaite and McKinley [8] the current version developed by Christopher et al. is described as a micro-electromechanical system oscillatory shear microrheometer (MEMS- $\mu \mathrm{R}$ ) [12]. The device uses a one-dimensional nanopositioner stage, which 


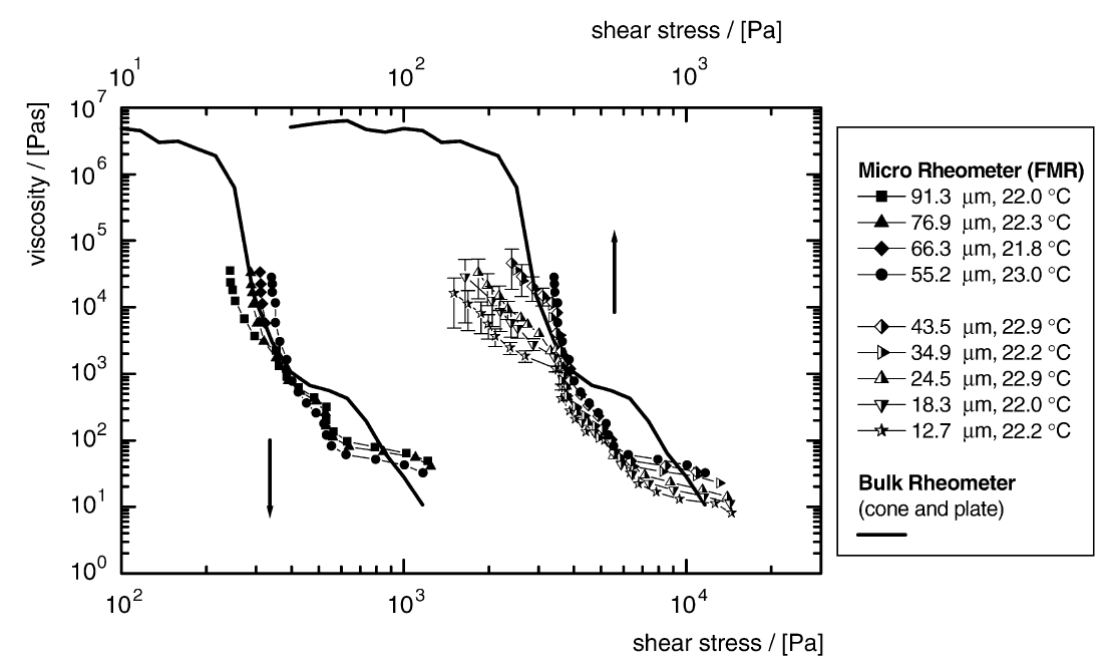

Figure 7: Example of viscosity curves for skin cream confined in gaps ranging from 13 to $91 \mu \mathrm{m}$, measured in the FMR. Bulk measurements using a cone and plate geometry are added as a solid line. For ease of visualization the results are split in two groups with the upper and lower abscissa [19].

applies a linear strain to the sample. The gap height of $1-10 \mu \mathrm{m}$ between the shearing surfaces is adjusted by using different polymer spacers. Oscillatory shearing deformation are induced by Joule heating that drives the holding beams to expand and buckle. By recording the motion with a reflected light microscope, both amplitude and phase difference can be used to determine dynamic moduli at an applied frequency.

Analogous to the errors in conventional rheometers that are used at narrow gap spacings, misalignment is the principal source of inaccuracy in sliding plate microrheometry. A tilt angle accuracy has been reported of $0.003^{\circ}$ for the FMR $[6,17,18]$ and $0.0075^{\circ}$ for the biaxial shearing cell [52]. For the MEMS- $\mu \mathrm{R}$, a compliance in the actuated stage allows for an upward displacement caused by surface tension [12]. Further gap errors can be caused by dust particles $(\sim 5 \mu \mathrm{m})$ that are trapped in the narrow gap [17] or the non-flatness of the surfaces $[12,17]$. Secondary flows is on the order of the gap height and can be neglected if the contact area is sufficiently large [17].

Sliding plate rheometers have been deployed successfully in several recent studies. Confinement effects and yielding phenomena in microstructured emulsions [17] and suspensions [52] have been investigated, as well as steady shear rheology on thin films to detect the onset of non-Newtonian behavior at high shear rates [18]. An example of confinement dependent microrheology of a complex fluid determined with a sliding plate rheometer, is given in figure 7. Because of the simple planar Couette flow, also slip phenomena and slip velocities (e.g. for yield stress fluids [14] or polymer melts [36]) have been probed.

\subsection{Microfluidics}

In general, microfluidics is considered to be the handling of small fluid volumes in geometries that have a characteristic size on the order of $0.1-100 \mu \mathrm{m}$. For microrheometry in the most simple form a microfluidic channel resembles a miniaturized version of a capillary rheometer in which the pressure drop over a channel section is related to the stress in the fluid, and the volumetric flow rate to the shear rate in the channel. For the general measuring principles of a capillary rheometer we refer at this point to standard textbooks [58]. In comparison to bulk capillary rheometers microfluidic rheometers as the commercial one depicted in figure 8 have several valuable features: low Reynolds number flows, high shear rates and small sample volumes $[11,42]$. In addition to this also the relative easy and cheap production of microfluidic chips has spawned a number of different designs of microfluidic (micro-)rheometers. Although there is a significant number of design parameters that vary between different devices, the general concept is the same. The channel geometry can be slit shaped [11, 27, 38, 49, 53, 75, 84, 88], square [42, 70] or a combination of a rectangular and a cylindrical shape [22]. The flow in the channels is either generated under a constant flow rate $[22,42,75,84]$ or a constant pressure $[27,53]$. The pressure drop over the 


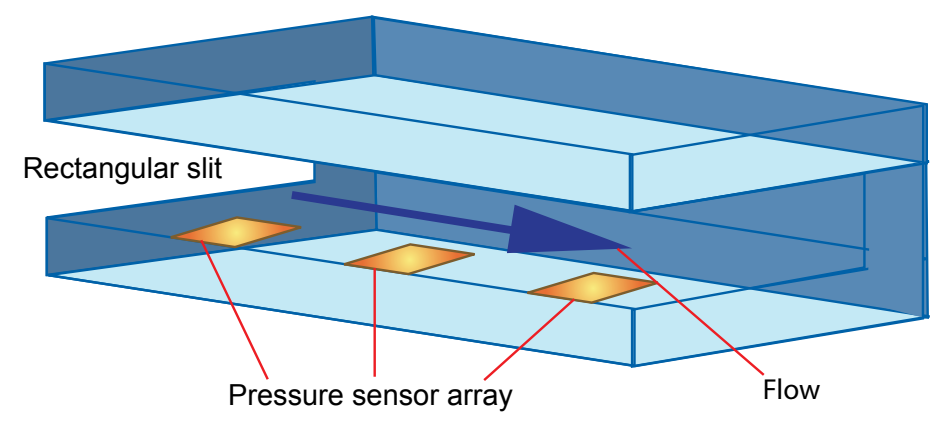

Figure 8: Schematic of the $m$-VROC microcapillary rheometer from Rheosense, Inc.

channel is measured by local pressure sensors in the channel walls [22, 75], inline with the channel [27, 42] or with pressure sensors in microcavities linked to the channel $[11,49,70,88]$. If the flow rate is not directly set, in some devices the flow rate is also monitored e.g. by recording the position of a fluid interface as a function of time [53], by electronic droplet sensing [88] or simply with a balance [27].

The limits of the microfluidic devices for microrheological measurements depends on their design. For channel heights typically on the order of $10-100 \mu \mathrm{m}[11,22,38,42,70,75]$ shear rates up to $10^{4}-10^{6} \mathrm{~s}^{-1}$ are achieved $[42,49,70]$. The measurable stress is restricted by either the measurement range of the pressure sensors or by the bonding strength of the device for which upper pressure limits on the order of $10^{6} \mathrm{~Pa}$ are reported [11].

Similar to other microrheology techniques, microfluidics is subject to several error sources. The above discussed viscous heating, inertia and turbulence can generally be neglected due to low Nahme and Reynolds numbers. However, for microfluidic rheometry three other sources for errors are discussed. Any deviation of the desired cross-section of the channels from the nominal values will affect the actual shear rate in the channel for a given flow rate. In particular local variations in the channel width and surface structure can significantly alter the flow profile [75]. Secondly, the application of constant flow rate in microfluidic devices is challenging [75]. Finally, similar to measurements in a regular capillary rheometer also the flow in microfluidic channels is affected by entrance and exit effects that need to be corrected for for example via a Bagley correction $[42,58]$

Microfluidic devices have been used extensively by i.a. the groups of Burns [53, 88] and McKinley $[38,75,84]$ due to the relatively low cost to produce and ease of operation [42]. The geometrical resemblance to many processing or usage conditions, the fact that no shear history is established on the fluid (only fresh material flows through the channels) and the advantage that the fluid has no free surface area (which prevents edge failure) all contribute to its success. Research on non-linear dynamics of semidilute aqueous polymer solutions uses microfabricated contraction-expansion geometries to identify the flow regimes and vortex growth mechanisms [49].

\subsection{Piezo vibrators}

Miniaturized vibrating sensors have been used successfully in rheological investigations, in particular developed by the groups of Pechold and Jakoby. Several types of micromachined vibrating actuators are suitable for the simultaneous measurement on the micro scale of viscosity and density as well as the storage and loss moduli, $G^{\prime}$ and $G^{\prime \prime}[30,31,44,51,78-80,91]$. The devices in this category can be grouped based on the phenomenon that is used to induce motion in the actuator (e.g. piezoelectric effect $[20,30,31,44,51,59,80,91]$, electromagnetic-acoustic effect $[78,79])$ and on the type of motion (e.g. axial [51, 91], shear [20, 30,31, 44, 59, 78, 79] or bending vibrations [80]). Furthermore, these vibrators can either be immersed in a liquid, have the sample deposited on top of the vibrator, or have the sample bounded by two surfaces. In the first case, as shown in figure 9 , only a microscale layer gets probed by the actuator (the thickness of the layer depends on the propagation depth of the shear wave [39]) while both latter cases measure a thin film that may confine the fluids microstructure.

Jakoby et al. outline several types of piezo vibrators [39]. Two common types that have been successfully implemented in rheological characterizations are thickness shear mode resonators and magnetic 


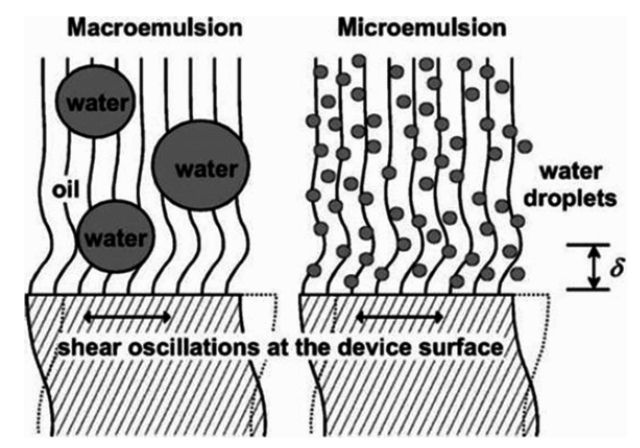

Figure 9: Illustration of the shear wave propagation (represented by the decay length of the shear wave $\delta$ ) through a fluid in contact with a TSM resonator [39]. The high frequencies employed in these devices, lead to only a small (micrometer) penetration depth of the shear wave. For macroemulsions with a characteristic size of the droplets larger than $\delta$, the sensor will not be affected by the presence of the droplets and only probe the viscosity of the continuous phase.

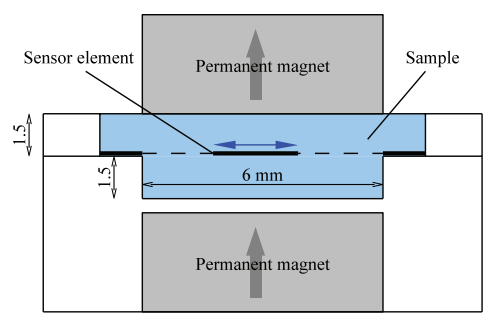

(a) Cross-sectional view

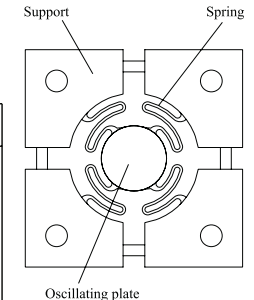

(b) Resonator elemen

Figure 10: Illustration of a plate resonator immersed in the fluid [79]. The metallic resonator is centered between the bottom housing and the top magnet. Vibrations are excited by Lorentz-forces in combination with the perpendicularly oriented magnetic field. As a result an electric field is generated in the moving plate and detected in a second loop.

acoustic resonators. Thickness shear mode resonators (TSM) use a quartz disk with electrodes on both sides. By imposing an $\mathrm{AC}$ voltage over these electrodes, vibrations are generated in the disk due to the piezoelectric effect. When the disk is immersed in a liquid, the viscosity and density can be determined since the liquid adds an additional mass and enhances viscous damping of the device. TSM resonators can be manufactured to excite axial, shear or bending vibrations. Like vibration due to the piezoelectric effect, acoustic waves can be generated with magnetic acoustic resonator sensors (MARS), as shown in figure 10. When a static magnetic field is placed next to electrically conducting disks, Lorentz forces generate various mechanical vibration modes. The interaction of the vibrating structure with the fluid is similar to the response in a TSM resonator [39].

The operational window depends on the specific device and cannot be generalized as such. Nevertheless, several common ranges can be provided. Due to the integrated nature of these systems, extreme operating conditions are possible: e.g. high temperatures, broad range of $\mathrm{pH}$-values and high pressures. Compared to conventional rheometers that operate in a frequency range of $10^{-5}-10^{2} \mathrm{~Hz}$, the micromachined devices excite vibrations between $10^{-1}$ and $10^{5} \mathrm{~Hz}$ and can thus also be utilized as high-frequency rheometers (in addition to their capability to probe small length scales. For systems that investigate confined samples, gaps in the range of 5-200 $\mu \mathrm{m}$ have been reported [91]. Applying this technique to do rheological measurements involves correlating the interaction of the sensor and the sample to rheological characteristics. However, this relation is not trivial [80] and requires most of the time the calibration with fluids of known properties [79, 80] to characterize the damped vibration of the surfaces.

Piezo vibrators (PVs) have provided microrheological insight in a number of recent studies. They can monitor crystallization through the viscosity of the reaction mixture during zeolite crystal formation in thin layers and fluid volumes [30]. In this application it is key that the device presents an in situ, real-time and non-destructive measurement. In other investigations, PVs have been used to complement measurements in a conventional rheometer to expand the frequency range in the determination of dynamic 


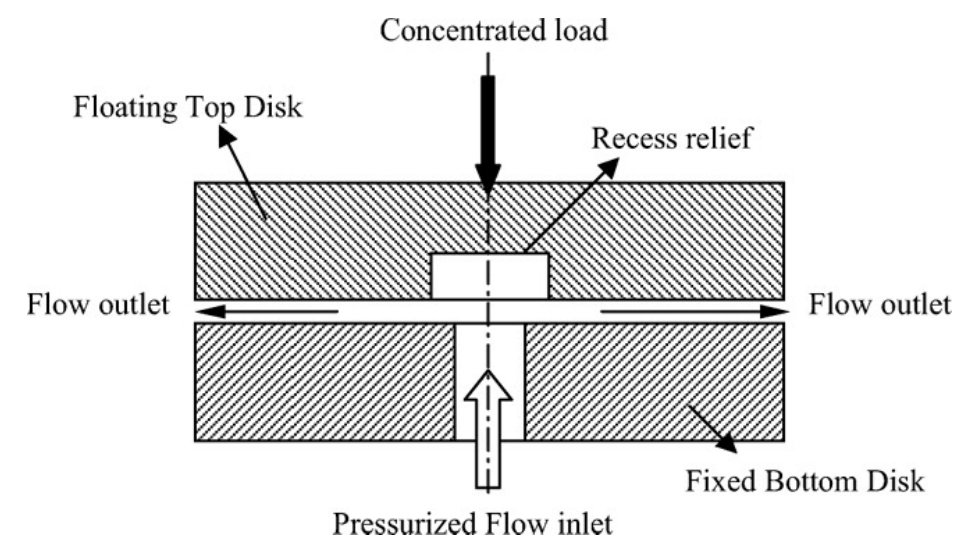

Figure 11: Cross sectional illustration of the PDRFM by Xie et al. [97]

moduli $[43,51,67,68,71,92]$ and to access the linear viscoelastic properties of fluids that exhibit short relaxation times. Relaxation times as low as $10 \mu$ s can be studied, which are comparable to jetting processes $(30 \mu \mathrm{s})$ [91]. Furthermore, piezo axial and rotary vibrators have been used to study in situ the rheology of drying, microscopically thin films [73]. Collin and Martinoty have used a rheometric device that relies on the piezoelectric effect to study the effect of confinement on the complex moduli of liquid-crystal polymers and on polymer melts. They reported a controversial observation for a micrometer confinement dependent transition from a liquid to a gel-like response for thin samples $(<20 \mu \mathrm{m})$ $[20,59]$. The discussion of this result sparked a discussion on the possible error sources for non-optical microrheology as for example pointed out by McKenna [61].

\subsection{Radial channel flows}

The microrheological investigation techniques with rotational or sliding plate rheometers described above have with their misalignment error a distinct disadvantage during a shearing deformation. In order to accommodate this issue, radial flow rheometers have been designed. The constant velocity squeeze flow rheometer (CVSFR) of Yan et al. [98] uses well aligned, roughened parallel plates that squeeze out the fluid in between with velocities in the range of $50-600 \mathrm{um} \mathrm{s}^{-1}$ while monitoring the normal stress with a piezoelectric force transducer. The device can operate down to confinement levels of $6-30 \mu \mathrm{m}$. Rheological parameters are calculated from the normal stress and the velocity (e.g. via the Stefan equation for Newtonian fluids) [98].

In the self-aligned pressure-driven radial flow microrheometer (PDRFM) of Xie et al. [97] as shown in figure 11, a pair of flat concentric disks is used where the upper surface can bob freely on the fluid film. The latter is established by pressurizing the fluid into the gap between the disks through an inlet channel at the center of the bottom surface. The upper disk is pushed down with a constant load and lubrication flows cause a constant gap that can be adjusted via the applied load. The fluid film thickness is measured and gaps are reported over a range of $55-152 \mu \mathrm{m}$. A maximum steady state tilt angle was measured to be $1.2 \times 10^{-5} \mathrm{rad}$ [97].

Willenbacher and co-workers have similarly used a ring-slit die in a capillary viscometer to study the flow-induced aggregation $[29,32,50]$. The piston of the viscometer moves at a constant velocity in the range of $1-990 \mathrm{~mm} \mathrm{~min}^{-1}$ and forces the sample to flow at a constant flow rate through a slit with an adjustable height between 10 and $30 \mu \mathrm{m}$. In this setup, the pressure is measured with a transducer, located above the die (as shown in figure 12).

\section{Conclusions}

The versatility of microrheological techniques has instigated a change in the characterization of complex fluids. Whereas measurements of the bulk rheology have played a dominant role in the past, it has become apparent that, depending on the usage or processing conditions, this is insufficient to unravel all the phenomena encountered in real-life applications as well as in fundamental investigations. Microrheology 


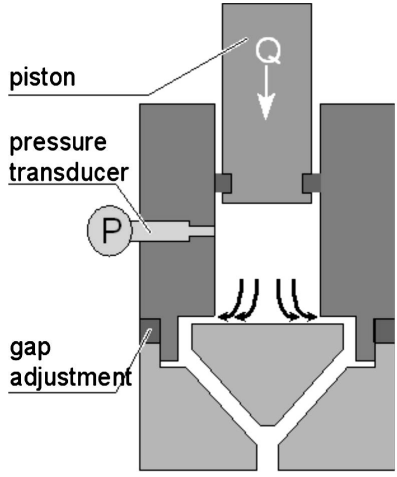

(a) ring-slit device

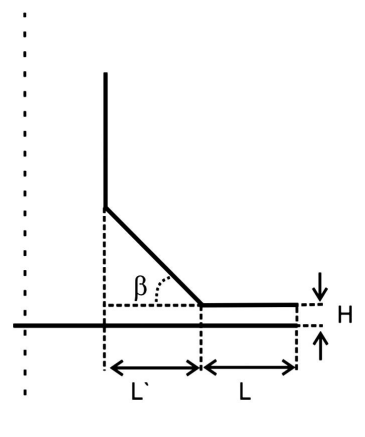

(b) slit entrance geometry

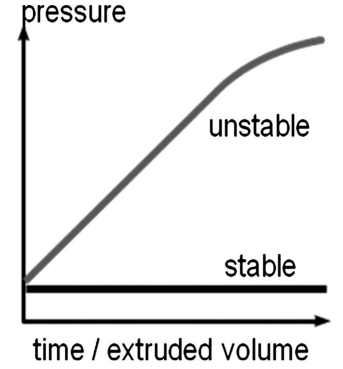

(c) extruded volume vs. pressure

Figure 12: Illustration of the ring-slit device [32].

is one additional tool for more specific investigations. Whereas optical microrheology focuses on local dynamics and properties of a sample, the non-optical techniques discussed here allow to probe bulk samples while taking one or more relevant dimensions to the microscale. This can be a confinement of the sample: in a comparison between two different types of mayonnaise, Clasen et al. found no difference in the bulk rheology of natural and fat free types using a conventional cone and plate rheometer, concluding that both were designed to have the same macroscopic shear flow behavior. However, utilizing a sliding plate rheometer on a microscopic scale, the measurements revealed a difference in the interaction of the microstructure with the confining geometry, possibly accounting for the difference in texture and mouthfeel [13]. Similarly the use of microrheology has been advocated by Stokes and coworkers, who state that confined Carbopol suspensions reveal a solid-like behavior of the gel with decreasing gap [24]. Other applications of non-optical microrheology include the improved investigation of boundary layer effects of bulk samples as slip [14], stick, [17] or yield layers [19]. In addition to rheological effects that are directly probed by microrheological measurements, other perks have equally been illustrated. For instance, the rheology of minute amounts of spider silk or drosera plant mucus has been investigated at large deformation rates, comparable to the strains encountered in vivo [28]. As a matter of fact, the low availability of fluid extracted from the spider's silk gland would render characterization using a conventional bulk rheometer impossible [45].

There is no straightforward choice from the broad range of non-optical microrheological techniques. The type of sample (e.g. availability, interfacial properties) and the desired information (e.g. properties akin to a certain process, fundamental properties for modeling) play a crucial role in this decision. For most investigations however, microrheology will complement measurements of bulk properties.

\section{Acknowledgement}

The authors would like to acknowledge the research foundation Flanders (FWO, Project No. G.0543.10N) for financial support.

\section{References}

[1] Anastasiadis, S. H., and Hatzikiriakos, S. G. The work of adhesion of polymer/wall interfaces and its association with the onset of wall slip. Journal of Rheology 42, 4 (July 1998), 795-812.

[2] Andablo-Reyes, E., de Vicente, J., And Hidalgo-Álvarez, R. On the nonparallelism effect in thin film plateplate rheometry. Journal of Rheology 55, 5 (July 2011), 981-986.

[3] Andablo-Reyes, E., Hidalgo-Álvarez, R., And de Vicente, J. A method for the estimation of the film thickness and plate tilt angle in thin film misaligned plateplate rheometry. Journal of Non-Newtonian Fluid Mechanics 165, 19-20 (Oct. 2010), 1419-1421. 
[4] Andablo-Reyes, E., Hidalgo-Álvarez, R., and de Vicente, J. Controlling friction using magnetic nanofluids. Soft Matter 7, 3 (2011), 880-883.

[5] BAIK, S. J. Microrheological Investigation of Sheared Thin Films with Complex Structure. PhD thesis, KU Leuven, 2011.

[6] Baik, S. J., Moldenaers, P., And Clasen, C. A sliding plate microgap rheometer for the simultaneous measurement of shear stress and first normal stress difference. The Review of scientific instruments 82, 3 (Mar. 2011), 035121.

[7] Barnes, H. A. A review of the slip (wall depletion) of polymer solutions, emulsions and particle suspensions in viscometers: its cause, character, and cure. Journal of Non-Newtonian Fluid Mechanics 56, 3 (Mar. 1995), 221-251.

[8] Braithwaite, G., And McKinley, G. Microrheometry for studying the rheology and dynamics of polymers near interfaces. Appl. Rheol, February (1999), 27-33.

[9] Buscall, R. Letter to the Editor: Wall slip in dispersion rheometry. Journal of Rheology 54, 6 (Oct. 2010), 1177-1183.

[10] Cheikn, C., And Koper, G. Stick-Slip Transition at the Nanometer Scale. Physical Review Letters 91, 15 (Oct. 2003), 156102.

[11] Chevalier, J., And Ayela, F. Microfluidic on chip viscometers. The Review of scientific instruments 79, 7 (July 2008), 076102.

[12] Christopher, G. F., Yoo, J. M., Dagalakis, N., Hudson, S. D., and Migler, K. B. Development of a MEMS based dynamic rheometer. Lab on a chip 10, 20 (Oct. 2010), 2749-57.

[13] Clasen, C. A new Flexure-based Microgap Rheometer (FMR) Case Study : Microrheology of Mayonnaise. Rheo-Future Young Scientist Award 2002 (2002).

[14] Clasen, C. Determining the true slip of a yield stress material with a sliding plate rheometer. Rheologica Acta 51, 10 (Aug. 2012), 883-890.

[15] Clasen, C. A self-aligning parallel plate (SAPP) fixture for tribology and high shear rheometry. Rheologica Acta 52, 3 (Feb. 2013), 191-200.

[16] Clasen, C. High shear rheometry using hydrodynamic lubrication flows. Journal of Rheology 57, 1 (Nov. 2013), 197-221.

[17] Clasen, C., Gearing, B. P., and McKinley, G. H. The flexure-based microgap rheometer (FMR). Journal of Rheology 50, 6 (2006), 883-905.

[18] Clasen, C., Kavehpour, H. P., and Mckinley, G. H. Bridging Tribology and Microrheology of Thin Films. Rheologica Acta 20, 4 (2010), 1-13.

[19] Clasen, C., And McKinley, G. H. Gap-dependent microrheometry of complex liquids. Journal of Non-Newtonian Fluid Mechanics 124, 1-3 (Dec. 2004), 1-10.

[20] Collin, D., And Martinoty, P. Dynamic macroscopic heterogeneities in a flexible linear polymer melt. Physica A: Statistical Mechanics and its Applications 320 (Mar. 2003), 235-248.

[21] Connelly, R. W., And Greener, J. High-Shear Viscometry with a Rotational Parallel-Disk Device. Journal of Rheology 29, 2 (Apr. 1985), 209-226.

[22] Craven, T. J., Rees, J. M., and Zimmerman, W. B. Pressure sensor positioning in an electrokinetic microrheometer device: simulations of shear-thinning liquid flows. Microfluidics and Nanofluidics 9, 2-3 (Feb. 2010), 559-571.

[23] Crawford, N. C., Williams, S. K. R., Boldridge, D., and Liberatore, M. W. Shear thickening of chemical mechanical polishing slurries under high shear. Rheologica Acta 51, 7 (May 2012), 637-647. 
[24] Davies, G., And Stokes, J. Thin film and high shear rheology of multiphase complex fluids. Journal of Non-Newtonian Fluid Mechanics 148, 1-3 (Jan. 2008), 73-87.

[25] Davies, G. A., And Stokes, J. R. On the gap error in parallel plate rheometry that arises from the presence of air when zeroing the gap. Journal of Rheology 49, 4 (July 2005), 919-922.

[26] Dontula, P., Macosko, C. W., and Scriven, L. E. Does the Viscosity of Glycerin Fall at High Shear Rates? Industrial \& Engineering Chemistry Research 38, 4 (Apr. 1999), 1729-1735.

[27] Erickson, D., Lu, F., Li, D., White, T., and Gao, J. An experimental investigation into the dimension-sensitive viscosity of polymer containing lubricant oils in microchannels. Experimental Thermal and Fluid Science 25, 8 (Feb. 2002), 623-630.

[28] Erni, P., Varagnat, M., Clasen, C., Crest, J., and McKinley, G. H. Microrheometry of sub-nanolitre biopolymer samples: non-Newtonian flow phenomena of carnivorous plant mucilage. Soft Matter 7, 22 (2011), 10889-10898.

[29] Ettl, R., Schädler, V., And Willenbacher, N. Runnability and flow-induced aggregation of paper coating suspensions. Nordic Pulp and Paper Research Journal 15, 5 (2000), 509-514.

[30] Follens, L. R. A., Reichel, E. K., Riesch, C., Vermant, J., Martens, J. A., Kirschhock, C. E. A., And Jakoby, B. Viscosity sensing in heated alkaline zeolite synthesis media. Physical chemistry chemical physics 11, 16 (Apr. 2009), 2854-2857.

[31] Fritz, G., Pechhold, W., Willenbacher, N., and Wagner, N. J. Characterizing complex fluids with high frequency rheology using torsional resonators at multiple frequencies. Journal of Rheology 47, 2 (Feb. 2003), 303-319.

[32] Georgieva, K., Dijkstra, D. J., Fricke, H., and Willenbacher, N. Clogging of microchannels by nano-particles due to hetero-coagulation in elongational flow. Journal of colloid and interface science 352, 2 (Dec. 2010), 265-277.

[33] Giacomin, A. J., Samurkas, T., and Dealy, J. M. A novel sliding plate rheometer for molten plastics. Polymer Engineering and Science 29, 8 (Apr. 1989), 499-504.

[34] Gillies, G., Prestidge, C. A., And Attard, P. An AFM Study of the Deformation and Nanorheology of Cross-Linked PDMS Droplets. Langmuir 18, 5 (Mar. 2002), 1674-1679.

[35] Gong, J. P., Kagata, G., and Osada, Y. Friction of Gels. 4. Friction on Charged Gels. Journal of Physical Chemistry B 103, 29 (July 1999), 6007-6014.

[36] Hatzikiniakos, S. G. Wall slip of molten polymers. Progress in Polymer Science 37, 4 (Apr. 2012), 624-643.

[37] Henson, D. J. Effect of gap on the viscosity of monodisperse polystyrene melts: Slip effects. Journal of Rheology 39, 2 (Mar. 1995), 359-373.

[38] Jaishankar, A., Sharma, V., And McKinley, G. H. Interfacial viscoelasticity, yielding and creep ringing of globular proteinsurfactant mixtures. Soft Matter 7, 17 (Aug. 2011), 7623-7634.

[39] Jakoby, B., Beigelbeck, R., Keplinger, F., Lucklum, F., Niedermayer, A., Reichel, E. K., Riesch, C., Voglhuber-Brunnmaier, T., And Weiss, B. Miniaturized sensors for the viscosity and density of liquids-performance and issues. IEEE transactions on ultrasonics, ferroelectrics, and frequency control 57, 1 (Jan. 2010), 111-120.

[40] Kagata, G., Gong, J. P., And Osada, Y. Friction of Gels. 6. Effects of Sliding Velocity and Viscoelastic Responses of the Network. Journal of Physical Chemistry B 106, 18 (May 2002), 45964601.

[41] Kagata, G., Gong, J. P., and Osada, Y. Friction of Gels. 7. Observation of Static Friction between Like-Charged Gels. Journal of Physical Chemistry B 107, 37 (Sept. 2003), 10221-10225. 
[42] Kang, K., Lee, L. J., And Koelling, K. W. High shear microfluidics and its application in rheological measurement. Experiments in Fluids 38, 2 (Jan. 2005), 222-232.

[43] Kheirandish, S., Gubaydullin, I., And Willenbacher, N. Shear and elongational flow behavior of acrylic thickener solutions. Part II: effect of gel content. Rheologica Acta 48, 4 (Nov. 2008), $397-407$.

[44] Kirschenmann, L., And Pechhold, W. Piezoelectric Rotary Vibrator (PRV) a new oscillating rheometer for linear viscoelasticity. Rheologica Acta 41, 4 (Apr. 2002), 362-368.

[45] Kojić, N., Bico, J., Clasen, C., And McKinley, G. H. Ex vivo rheology of spider silk. Journal of experimental biology 209, 21 (Nov. 2006), 4355-4362.

[46] Koliandris, A.-L., Morris, C., Hewson, L., Hort, J., Taylor, A. J., And Wolf, B. Correlation between saltiness perception and shear flow behaviour for viscous solutions. Food Hydrocolloids 24, 8 (Nov. 2010), 792-799.

[47] Kramer, J., Uhl, J. T., and Prud'Homme, R. K. Measurement of the viscosity of guar gum solutions to $50,000 \mathrm{~s}^{-1}$ using a parallel plate rheometer. Polymer Engineering and Science 27, 8 (Apr. 1987), 598-602.

[48] Kulicke, W. M., And Porter, R. S. Relation between steady shear flow and dynamic rheology. Rheologica Acta 19, 5 (Sept. 1980), 601-605.

[49] Lanzaro, A., And YuAn, X.-F. Effects of contraction ratio on non-linear dynamics of semi-dilute, highly polydisperse PAAm solutions in microfluidics. Journal of Non-Newtonian Fluid Mechanics 166, 17-18 (Sept. 2011), 1064-1075.

[50] Lee, A., Sudau, K., Ahn, K. H., Lee, S. J., And Willenbacher, N. Optimization of Experimental Parameters to Suppress Nozzle Clogging in Inkjet Printing. Industrial E Engineering Chemistry Research 51, 40 (Oct. 2012), 13195-13204.

[51] Li, Z., Yuan, X.-F., Haward, S. J., Odell, J. A., and Yeates, S. Non-linear dynamics of semi-dilute polydisperse polymer solutions in microfluidics: A study of a benchmark flow problem. Journal of Non-Newtonian Fluid Mechanics 166, 16 (Sept. 2011), 951-963.

[52] Lin, N. Y. C., Cheng, X., And Cohen, I. Biaxial shear of confined colloidal hard spheres: the structure and rheology of the vorticity-aligned string phase. Soft Matter 10, 12 (Feb. 2014), 1969-1976.

[53] Livak-Dahl, E., Lee, J., And Burns, M. A. Nanoliter droplet viscometer with additive-free operation. Lab on a chip 13, 2 (Jan. 2013), 297-301.

[54] Luengo, G., Schmitt, F.-J., Hill, R., And Israelachvili, J. Thin Film Rheology and Tribology of Confined Polymer Melts: Contrasts with Bulk Properties. Macromolecules 30, 8 (Apr. 1997), $2482-2494$.

[55] Maciel, A., Salas, V., Soltero, J. F. A., Guzmán, J., and Manero, O. On the wall slip of polymer blends. Journal of Polymer Science Part B: Polymer Physics 40, 4 (Feb. 2002), 303-316.

[56] Mackay, M. E., And Henson, D. J. The effect of molecular mass and temperature on the slip of polystyrene melts at low stress levels. Journal of Rheology 42, 6 (Nov. 1998), 1505.

[57] MacKintosh, F., And Schmidt, C. Microrheology. Current Opinion in Colloid E Interface Science 4, 4 (Aug. 1999), 300-307.

[58] Macosko, C. W. Rheology: Principles, Measurements and Applications. Wiley-VCH, Inc., 1994.

[59] Martinoty, P., Hilliou, L., Mauzac, M., Benguigui, L., And Collin, D. Side-Chain LiquidCrystal Polymers: Gel-like Behavior below Their Gelation Points. Macromolecules 32, 6 (Mar. 1999), 1746-1752. 
[60] Mateyawa, S., Xie, D. F., Truss, R. W., Halley, P. J., Nicholson, T. M., Shamshina, J. L., Rogers, R. D., Boenm, M. W., and MeNally, T. Effect of the ionic liquid 1-ethyl-3methylimidazolium acetate on the phase transition of starch: dissolution or gelatinization? Carbohydrate polymers 94, 1 (Apr. 2013), 520-530.

[61] McKenna, G. B. Commentary on rheology of polymers in narrow gaps. The European physical journal E 19, 1 (Jan. 2006), 101-108.

[62] Meeker, S. P., Bonnecaze, R. T., and Cloitre, M. Slip and flow in pastes of soft particles: Direct observation and rheology. Journal of Rheology 48, 6 (Nov. 2004), 1295.

[63] Mooney, M. Explicit Formulas for Slip and Fluidity. Journal of Rheology 2, 2 (Oct. 1931), 210-222.

[64] Moschakis, T. Microrheology and particle tracking in food gels and emulsions. Current Opinion in Colloid \& Interface Science 18, 4 (Aug. 2013), 311-323.

[65] Mriziq, K. S., Dai, H. J., Dadmun, M. D., Jellison, G. E., and Cochran, H. D. Highshear-rate optical rheometer. Review of Scientific Instruments 75, 6 (May 2004), 2171-2176.

[66] Mukhopadhyay, A., and Granick, S. Micro- and nanorheology. Current Opinion in Colloid $\mathcal{E}^{3}$ Interface Science 6, 5-6 (Nov. 2001), 423-429.

[67] Oelschlaeger, C., Cota Pinto Coelho, M., and Willenbacher, N. Chain flexibility and dynamics of polysaccharide hyaluronan in entangled solutions: a high frequency rheology and diffusing wave spectroscopy study. Biomacromolecules 14, 10 (Oct. 2013), 3689-3696.

[68] Oelschlaeger, C., Schopferer, M., Scheffold, F., and Willenbacher, N. Linear-tobranched micelles transition: a rheometry and diffusing wave spectroscopy (DWS) study. Langmuir : the ACS journal of surfaces and colloids 25, 2 (Jan. 2009), 716-723.

[69] Olagunju, D. O. Effect of free surface and inertia on viscoelastic parallel plate flow. Journal of Rheology 38, 1 (Jan. 1994), 151-168.

[70] Pan, L., And Arratia, P. E. A high-shear, low Reynolds number microfluidic rheometer. Microfluidics and Nanofluidics 14, 5 (Dec. 2012), 885-894.

[71] Pawelzyk, P., Mücke, N., Herrmann, H., and Willenbacher, N. Correction: Attractive Interactions among Intermediate Filaments Determine Network Mechanics In Vitro. PloS one 9, 4 (Jan. 2014), e93194.

[72] Peachey, J., Van Alsten, J., And Granick, S. Design of an apparatus to measure the shear response of ultrathin liquid films. Review of Scientific Instruments 62, 2 (Feb. 1991), 463-473.

[73] Pechhold, W., Mayer, U., Raju, G. B., and Gulllon, O. Piezo rotary and axial vibrator (PRAV) characterization of a fresh coating during its drying. Rheologica Acta 50, 3 (Jan. 2011), 221-229.

[74] Pfleiderer, P., Baik, S. J., Zhang, Z., Vleminckx, G., Lettinga, M. P., Grelet, E., Vermant, J., and Clasen, C. X-ray scattering in the vorticity direction and rheometry from confined fluids. The Review of scientific instruments 85, 6 (June 2014), 065108.

[75] Pipe, C. J., Majmudar, T. S., And McKinley, G. H. High shear rate viscometry. Rheologica Acta 47, 5-6 (Apr. 2008), 621-642.

[76] Pit, R., Hervet, H., And LÉger, L. Direct Experimental Evidence of Slip in Hexadecane: Solid Interfaces. Physical Review Letters 85, 5 (July 2000), 980-983.

[77] Pullarkat, P. A., Fernández, P. A., and Ott, A. Rheological properties of the Eukaryotic cell cytoskeleton. Physics Reports 449, 1-3 (Sept. 2007), 29-53.

[78] Reichel, E. K., Heinisch, M., Jakoby, B., Vermant, J., and Kirschhock, C. E. Viscoelasticity Sensor with Resonance Tuning and Low-Cost Interface. Procedia Engineering 25 (Jan. 2011), $623-626$. 
[79] Reichel, E. K., Vermant, J., Jakoby, B., and Kirschhock, C. E. Shear wave sensors for viscoelastic properties. Procedia Engineering 5 (Jan. 2010), 1316-1319.

[80] Riesch, C., Reichel, E. K., Keplinger, F., And Jakoby, B. Characterizing Vibrating Cantilevers for Liquid Viscosity and Density Sensing. Journal of Sensors 2008 (2008), 1-9.

[81] Rother, J., Nöding, H., Mey, I., And Janshoff, A. Atomic force microscopy-based microrheology reveals significant differences in the viscoelastic response between malign and benign cell lines. Open biology 4, 5 (May 2014), 140046.

[82] Schmatko, T., Hervet, H., And Leger, L. Friction and Slip at Simple Fluid-Solid Interfaces: The Roles of the Molecular Shape and the Solid-Liquid Interaction. Physical Review Letters 94, 24 (June 2005), 244501.

[83] Seth, J. R., Cloitre, M., and Bonnecaze, R. T. Influence of short-range forces on wall-slip in microgel pastes. Journal of Rheology 52, 5 (Sept. 2008), 1241-1268.

[84] Sharma, V., Jaishankar, A., Wang, Y.-C., And McKinley, G. H. Rheology of globular proteins: apparent yield stress, high shear rate viscosity and interfacial viscoelasticity of bovine serum albumin solutions. Soft Matter 7, 11 (May 2011), 5150-5160.

[85] Sochi, T. Slip at Fluid-Solid Interface. Polymer Reviews 51, 4 (Oct. 2011), 309-340.

[86] Sorbie, K. Depleted layer effects in polymer flow through porous media. Journal of Colloid and Interface Science 139, 2 (Oct. 1990), 299-314.

[87] Squires, T. M., And Mason, T. G. Fluid Mechanics of Microrheology. Annual Review of Fluid Mechanics 42, 1 (Dec. 2010), 413-438.

[88] Srivastava, N., And Burns, M. A. Electronic drop sensing in microfluidic devices: automated operation of a nanoliter viscometer. Lab on a chip 6, 6 (June 2006), 744-751.

[89] Stokes, J. R., Boenm, M. W., And Baier, S. K. Oral processing, texture and mouthfeel: From rheology to tribology and beyond. Current Opinion in Colloid E Interface Science 18, 4 (Aug. 2013), 349-359.

[90] Turian, R. M. Perturbation Solution of the Steady Newtonian Flow in the Cone and Plate and Parallel Plate Systems. Industrial \& Engineering Chemistry Fundamentals 11, 3 (Aug. 1972), 361368.

[91] Vadillo, D. C., Mathues, W., and Clasen, C. Microsecond relaxation processes in shear and extensional flows of weakly elastic polymer solutions. Rheologica Acta 51, 8 (July 2012), 755-769.

[92] Vesaratchanon, J. S., Takamura, K., and Willenbacher, N. Surface characterization of functionalized latexes with different surface functionalities using rheometry and dynamic light scattering. Journal of colloid and interface science 345, 2 (May 2010), 214-221.

[93] Waigh, T. A. Microrheology of complex fluids. Reports on Progress in Physics 68, 3 (Mar. 2005), $685-742$.

[94] WANG, S.-Q. Exploring molecular origins of sharkskin, partial slip, and slope change in flow curves of linear low density polyethylene. Journal of Rheology 40, 5 (Sept. 1996), 875-898.

[95] Weis, C., Natalia, I., And Willenbacher, N. Effect of weak attractive interactions on flow behavior of highly concentrated crystalline suspensions. Journal of Rheology 58, 5 (Sept. 2014), $1583-1597$.

[96] White, C. C., Vanlandingham, M. R., Drzal, P. L., Chang, N.-K., and Chang, S.H. Viscoelastic characterization of polymers using instrumented indentation. II. Dynamic testing. Journal of Polymer Science Part B: Polymer Physics 43, 14 (July 2005), 1812-1824.

[97] Xie, Z., Zou, Q., And Yao, D. Design and Verification of the Pressure-Driven Radial Flow Microrheometer. Tribology Transactions 51, 4 (July 2008), 396-402. 
[98] Yan, Y., Zhang, Z., Cheneler, D., Stokes, J. R., and Adams, M. J. The influence of flow confinement on the rheological properties of complex fluids. Rheologica Acta 49, 3 (Dec. 2009), $255-266$.

[99] Yoshimura, A., And Prud'Homme, R. K. Wall Slip Corrections for Couette and Parallel Disk Viscometers. Journal of Rheology 32, 1 (Jan. 1988), 53-67. 